\title{
Tectal gliomas: assessment of malignant progression, clinical management, and quality of life in a supposedly benign neoplasm
}

\author{
${ }^{*}$ Malte Mohme, MD, ${ }^{1}$ Friederike S. Fritzsche, MD, ${ }^{1}$ Klaus C. Mende, MD, ${ }^{1}$ Jakob Matschke, MD, ${ }^{2}$ \\ Ulrike Löbel, MD, ${ }^{3}$ Gertrud Kammler, MD, ${ }^{1}$ Manfred Westphal, MD, ${ }^{1}$ Pedram Emami, MD, MBA, ${ }^{1}$ and \\ Tobias Martens, MD'
}

Departments of ${ }^{1}$ Neurosurgery and ${ }^{3}$ Neuroradiology and ${ }^{2}$ Institute of Neuropathology, University Medical Center Hamburg-Eppendorf, Hamburg, Germany

\begin{abstract}
OBJECTIVE Tectal gliomas constitute a rare and inhomogeneous group of lesions with an uncertain clinical course. Because these supposedly benign tumors are frequently followed up by observation over many years, the authors undertook this analysis of their own case series in an effort to demonstrate that the clinical course is highly variable and that there is a potential for a progressive biology.

METHODS Clinical data analysis of 23 cases of tectal glioma (involving 9 children and 14 adults) was performed retrospectively. Radiographic data were analyzed longitudinally and MR images were evaluated for tumor volume, contrast enhancement, and growth progression. Quality of life was assessed using the EORTC BN20 and C30 questionnaires during follow-up in a subgroup of patients.

RESULTS The patients' mean age at diagnosis was 29.2 years. The main presenting symptom at diagnosis was hydrocephalus $(80 \%)$. Six patients were treated by primary tumor resection (26.1\%), 3 patients underwent biopsy followed by resection (13.1\%), and 3 patients underwent biopsy only $(13.1 \%)$. For additional treatment of hydrocephalus, 14 patients $(60.9 \%)$ received shunts and/or endoscopic third ventriculostomy. Radiographic tumor progression was observed in $47.9 \%$ of the 23 cases. The mean time between diagnosis and growth progression was 51.5 months, and the mean time to contrast enhancement was 69.7 months. Histopathological analysis was obtained in 12 cases (52.2\%), resulting in 5 cases of high-grade glioma ( 3 cases of glioblastoma multiforme [GBM], grade IV, and 2 of anaplastic astrocytoma, grade III), 5 cases of pilocytic astrocytoma, 1 diffuse astrocytoma, and 1 ganglioglioma. Malignant progression was observed in 2 cases, with 1 case progressing from a diffuse astrocytoma (grade II) to a GBM (grade IV) within a period of 13 years. Quality-of-life measurements demonstrated distinct functional deficits compared to a healthy sample as well as glioma control cohorts.
\end{abstract}

CONCLUSIONS Analysis of this case series shows that a major subpopulation of tectal gliomas show progression and malignant transformation in children as well as in adolescents. These tumors therefore cannot be considered inert lesions and require histological confirmation and close follow-up. Quality-of-life questionnaires show that tectal glioma patients might benefit from special psychological support in emotional, social, and cognitive functionality.

https://thejns.org/doi/abs/10.3171/2018.3.FOCUS1850

KEYWORDS tectal glioma; hydrocephalus; malignant transformation; quality of life; brain stem; progression

$\mathrm{F}$ REQUENTLY considered to be a benign subgroup of astrocytoma, ${ }^{3,25}$ gliomas of the tectum mesencephali represent a complex condition, often demanding multi-staged surgical management and long-term follow up after diagnosis. Even though mesencephalic lesions have been extensively discussed over the past decade, ${ }^{10,14,18}$ the process of growth progression and malignant transfor- mation seems to be underestimated in the current literature. Investigating growth progression as a parameter for a potentially complicated course and conceivably malignant transformation of prior benign tumors should lead to closer follow-up and a changing understanding of an assumed subgroup of tumors at risk for malignant transformation. In addition, as most of the case series discussed in the lit-

ABBREVIATIONS GBM = glioblastoma multiforme; $H G$ = high grade; $L G=$ low grade .

SUBMITTED January 31, 2018. ACCEPTED March 20, 2018.

INCLUDE WHEN CITING DOI: 10.3171/2018.3.FOCUS1850.

* M.M. and F.S.F. contributed equally to this work. 
erature are predominantly pediatric, ${ }^{3,8,25}$ there might be a difference in the tumor biology and the clinical course in adult patients compared to children. Histologically, these case series are made up of very few samples of low-grade astrocytomas (pilocytic astrocytoma, WHO grade I). Even in the cases of radiographic disease progression, further therapy like stereotactic radiation or chemotherapy was usually implemented without a histological diagnosis.

In general, tectal gliomas, including intrinsic diffuse astrocytomas or pilocytic astrocytoma, are assumed to have a favorable prognosis as they are mostly considered indolent lesions, especially in children., ${ }^{5,25}$ Occlusive hydrocephalus is most often the presenting symptom that requires surgical treatment. ${ }^{5,9,14}$ If the hydrocephalus is adequately treated, patients are typically followed up by MRI and clinical examination. Potential parameters to predict a progressive course have not yet been accurately described in the literature.

We present here a subgroup of patients whose tumors demonstrated growth progression and subsequent malignant transformation. Moreover, this article emphasizes the correlation between MRI findings, time to tumor progression, and histological diagnosis as well as the role of quality-of-life assessment in this patient group. Our analysis subsequentially focuses on the discussion of current clinical management guidelines for patients with tectal gliomas.

\section{Methods \\ Patients}

We performed a retrospective analysis on data from 23 patients with tumors of the tectum mesencephali treated at our institution from 1979 to 2016. Patients were identified from case records and surgical protocol archives. The diagnosis was verified by neuroradiological criteria and reports. We included all patients with radiographic presentation suspicious for a glioma with clear association to the tectum mesencephali. Patients with regressive lesions, indicative of inflammation, and patients with lesions that were histologically proven to be entities other than glioma were excluded. "Tectal" was defined for the purpose of this study as primarily emanating from the quadrigeminal plate or extending beyond it but with the primary mass in the tectum. Periaqueductal tumors incorporating the tectum were included. Pineal lesions, which frequently displace and distort the tectum, were not included, ${ }^{28}$ nor were diffuse intrinsic pontine gliomas (DIPGs), which may have upward extension into upper brainstem structures. In 12 cases, the patients responses to quality-of-life questionnaires (EORTC QLQ C30 rev. 3.0 and BN20) were documented for routine evaluation and quality assessment of health-related quality of life during clinical follow-up contacts. Data analysis was performed retrospectively on anonymized clinical data sets. The study was conducted in accordance with ethical guidelines of the Hamburg (Germany) ethics review board and with ethics guidelines of the Declaration of Helsinki.

\section{Radiographic Evaluation}

MRI contrast enhancement was analyzed on T1-weight- ed images obtained after administration of a contrast agent. In a subgroup of patients, the first imaging assessment was undertaken using CT, as MRI was not available at that time. Tumor size was calculated from measurements of axial and sagittal T2-weighted MR images. Tumor growth progression and occurrence of contrast enhancement over the disease course were evaluated by an independent neuroradiologist.

\section{Statistical Analysis}

Statistical analysis was performed using chi-square and Student t-tests in Microsoft Excel 2016 and IBM SPSS version 24. Significance was defined on a $95 \%$ confidence interval. Graphical analysis of growth progression and contrast enhancement parameters was performed using GraphPad Prism. The quality-of-life results were compared to published cohorts using the Student t-test. Differences in the survival analysis were calculated using a Mantel-Cox log-rank test.

\section{Results}

\section{Patient Cohort}

Twenty-three patients with suspected tectal glioma were identified. Their mean age at diagnosis was 29 years (range 1-69 years); 10 patients were under the age of 18 years at the time of initial diagnosis. The mean duration of follow-up was 73 months (range 7-429 months). Twelve patients $(52.2 \%)$ were male, and $11(47.8 \%)$ were female. Their primary signs and symptoms on admission were hydrocephalus (78\%), visual impairment (26\%), cephalgia (13\%), gait disturbances (8.7\%), facial dysesthesia (4.3\%), primary amenorrhea (4.3\%), epilepsy (8.7\%), and developmental delay (4.3\%). Patient demographic and clinical characteristics are summarized in Table 1 and Fig. 1.

\section{Magnetic Resonance Imaging}

In 7 cases $(30.4 \%)$, the tumors showed contrast enhancement on initial MRI. In 5 of the remaining cases ( $21.7 \%$ of the overall cohort), the tumors developed contrast enhancement during follow-up (example in Fig. 2A). Excluding patients with primary enhancement at initial diagnosis, the mean time to contrast enhancement was 127.2 months (SD 50.3 months, $\mathrm{p}<0.05$, Fig. 3A). In addition, 11 of $23(47.8 \%)$ patients showed signs of tumor progression (e.g., increase in size), based on T2-weighted MRI during the observation period (mean time to progression 87.5 months, SD 120.0 months, $\mathrm{p}<0.05)$. Within the limited group of patients with growth progression, primary contrast-enhancing tumors showed a faster time to progression (mean 35.33 months, SD 19.69 months) than tumors that developed enhancement after initial diagnosis (secondary enhancement; mean time to progression 136 months, SD 39.14 months), whereas both groups had faster time to growth progression than nonenhancing tumors did ( $p=0.024$, Mantel-Cox log-rank test, Fig. 3B).

Primary aqueduct stenosis was present in $19(82.6 \%)$ patients, resulting in hydrocephalus in 18 (78.3\%) cases. The tumor volume at diagnosis ranged from 0.1 to $49.1 \mathrm{~cm}^{3}$ (mean $6.2 \mathrm{~cm}^{3}$, SD $10.4 \mathrm{~cm}^{3}, \mathrm{p}<0.05$ ). As expected, patients with hydrocephalus had a significantly higher mean 
tumor volume than those without hydrocephalus (7.6 vs 1.5 $\left.\mathrm{cm}^{3}, \mathrm{p}<0.05\right)$.

\section{Treatment}

Nine patients $(39.1 \%, n=9 / 23)$ underwent tumor resection $(25.7 \%, \mathrm{n}=9 / 35,7$ subtotal, 2 gross total). Overall, 6 patients $(26.1 \%, n=6 / 23$ ) were biopsied ( 2 of those twice due to inconclusive histology), out of which 3 patients $(13.1 \%, n=3 / 23)$ received a biopsy followed by resection (histology: GBM, grade IV, in 2 cases; anaplastic astrocytoma, grade III, in 1 case) and 3 patients received a biopsy only. Histological analysis in the biopsy-only group showed 2 pilocytic astrocytomas, WHO grade I, and 1 anaplastic astrocytoma, WHO grade III, which was not amenable to resection and was treated with chemotherapy. In six patients $(26.1 \%, \mathrm{n}=6 / 23)$ a primary resection and histological confirmation was performed. About half of all patients $(47.8 \%, \mathrm{n}=11 / 23)$ did not receive surgical histological confirmation by biopsy or resection and were followed with regular MRI.

Eighteen patients had hydrocephalus at presentation. For the treatment of hydrocephalus, 2 patients $(8.7 \%, \mathrm{n}=$ $2 / 23$ ) received a cerebrospinal fluid shunt only, 8 patients underwent endoscopic third ventriculostomy only (34.8\%, $\mathrm{n}=8 / 23)$, and 4 patients $(17.4 \%, \mathrm{n}=4 / 23)$ underwent both endoscopic third ventriculostomy and ventriculoperitoneal shunting. Four patients did not need additional surgery for hydrocephalus after resection.

Two patients received combined radiochemotherapy after tumor resection, 1 patient underwent radiation therapy (without chemotherapy) postoperatively, and 2 received chemotherapy after biopsy.

Of those patients showing tumor progression, those who had undergone tumor resection showed progression at mean of 68.4 months after initial presentation (vs 103.5 months without resection, no significant difference). However, enhancement in progressing tumors was seen at a mean of 118.0 months in patients who were not initially treated with resection and 93.0 months in those who were (no significant difference). Patients whose tumors showed no contrast enhancement at initial presentation but showed enhancement on subsequent follow up demonstrated a delay in mean time to progression when they underwent tumor resection. Hydrocephalus occurred at a mean of 62.3 months in patients without tumor resection as compared with 143.0 months in patients with resection ( $\mathrm{n}=2$ vs $\mathrm{n}=$ $3, p=0.3)$. Similarly, the mean time to enhancement was 99.7 versus 168.5 months $(n=3$ vs $n=2, p=0.12)$. This supports the concept of early surgical intervention, as the patient course otherwise shows earlier progression.

Two patients $(8.7 \%)$ died after a mean of 14.6 years due to malignant transformation and rapid tumor growth (Fig. 1). In both cases, histological findings revealed glioblastoma multiforme (GBM) WHO grade IV. In 1 case (patient 2), 2 samples of the histological progress were available-one after initial biopsy (WHO grade II) and one after subtotal resection (WHO grade IV) - within the course of clinical progression (Fig. 1).

In the few cases in which subtotal tumor resection was performed, no major surgical complications were observed. Malfunction of ventriculoperitoneal shunts or in-
TABLE 1. Overview of demographic and clinical characteristics of the 23 patients included this study

\begin{tabular}{|c|c|}
\hline Characteristic & Value \\
\hline \multicolumn{2}{|l|}{ Age at diagnosis, yrs } \\
\hline Mean & 29.2 \\
\hline Range & $1-69$ \\
\hline \multicolumn{2}{|l|}{ Sex } \\
\hline Male & $12(52.2 \%)$ \\
\hline Female & $11(47.8 \%)$ \\
\hline \multicolumn{2}{|l|}{ Follow-up, mos } \\
\hline Mean & $73 \pm 93$ \\
\hline Range & $7-429$ \\
\hline Histological analysis performed & $12(52.2 \%)$ \\
\hline \multicolumn{2}{|l|}{ Tumor WHO grade } \\
\hline I & $6 / 12(50.0 \%)^{*}$ \\
\hline II & $1 / 12(8.3 \%)$ \\
\hline III & $2 / 12(16.7 \%)$ \\
\hline IV & $3 / 12(25.0 \%)$ \\
\hline Aqueduct stenosis & $19(82.6 \%)$ \\
\hline Hydrocephalus & $18(78.3 \%)$ \\
\hline \multicolumn{2}{|l|}{ Tumor volume, $\mathrm{cm}^{3}$} \\
\hline Mean & 6.2 \\
\hline Range & $0.1-49.1$ \\
\hline Contrast enhancement & $12 / 23(52.2 \%)$ \\
\hline Present on initial MRI & 7 \\
\hline Developed during follow-up & 5 \\
\hline \multicolumn{2}{|l|}{ Surgery } \\
\hline \multicolumn{2}{|l|}{ Hydrocephalus } \\
\hline Shunt only & $2(14.3 \%)$ \\
\hline ETV $\rightarrow$ shunt & $4(28.6 \%)$ \\
\hline ETV only & $8(57.1 \%)$ \\
\hline \multicolumn{2}{|l|}{ Tumor } \\
\hline Biopsy only & $3(25 \%)$ \\
\hline Biopsy $\rightarrow$ resection & $3(25 \%)$ \\
\hline Resection only & $6(50 \%)$ \\
\hline Adjuvant therapy & $4(17.4 \%)$ \\
\hline RT + chemo & $2(8.7 \%)$ \\
\hline RT only & $1(4.3 \%)$ \\
\hline Chemo only & $1(4.3 \%)$ \\
\hline
\end{tabular}

Chemo = chemotherapy; ETV = endoscopic third ventriculostomy; RT = radiotherapy.

* Includes 5 pilocytic astrocytomas and 1 ganglioglioma.

fections due to external ventricular drainage did not differ from the known complication rates for these procedures.

\section{Histology}

Histological findings were available for 12 cases, of which 7 were classified as low-grade (LG) tumors (5 pilocytic astrocytomas, grade I; 1 ganglioglioma, grade I; 1 diffuse astrocytoma, grade II) and 5 as high-grade (HG) tumors (3 GBMs, grade IV; and 2 anaplastic astrocytomas, grade III). Histological grades describe the last tested 


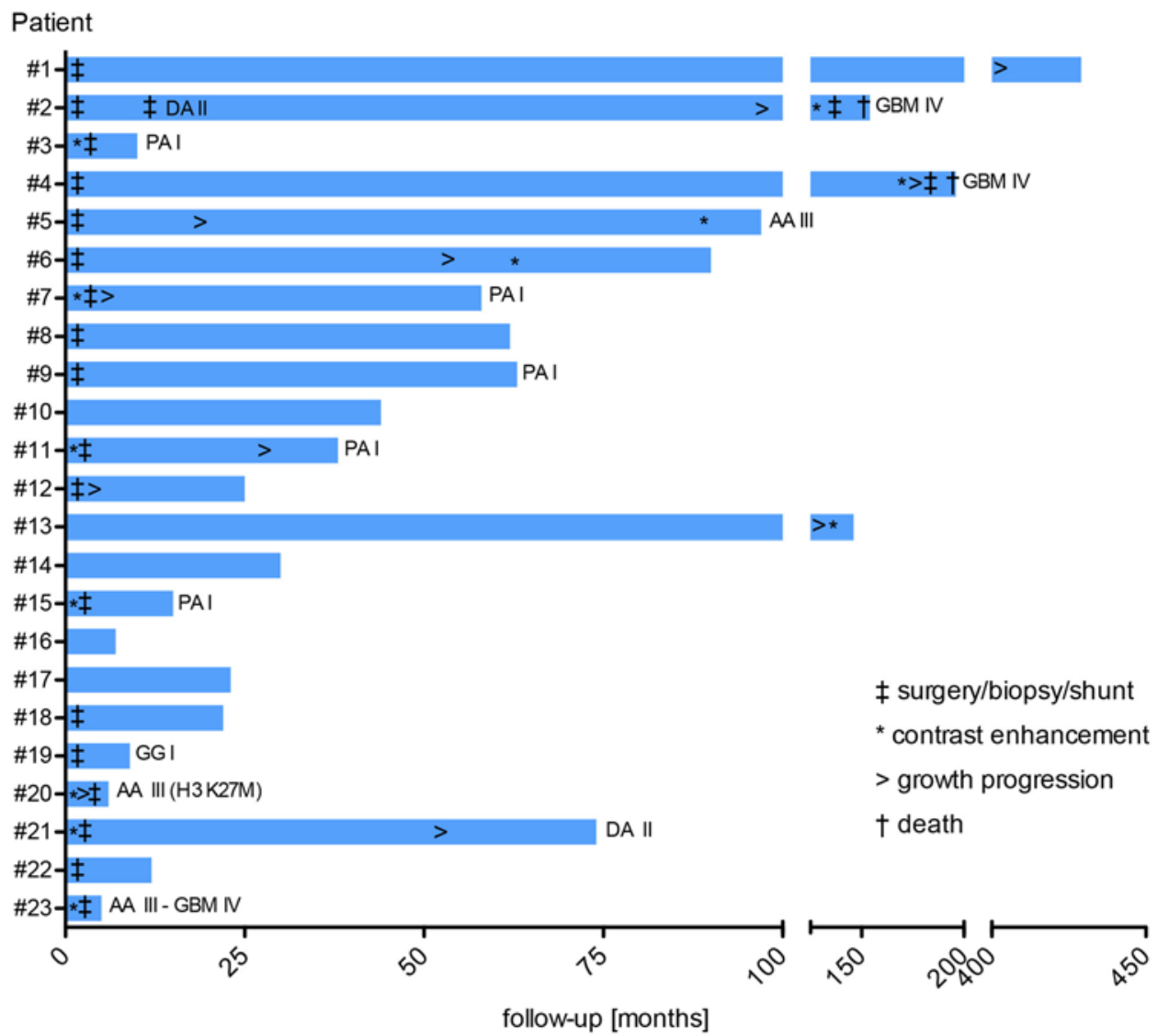

FIG. 1. Gantt plot depicting the individual follow-up of the 23 tectal glioma patients. Surgical interventions and MRI findings and patient death are marked as shown in the key. AA = anaplastic astrocytoma (WHO grade III); DA = diffuse astrocytoma (WHO grade II); GBM = glioblastoma multiforme (WHO grade IV); GG = ganglioglioma (WHO grade I); PA = pilocytic astrocytoma (WHO grade I).

tumor grade during the disease course. Interestingly, primary contrast enhancement was found in $5(71.4 \%)$ of 7 LG tumors and $2(40.0 \%)$ of 5 HG tumors (Table 1). Immunohistochemical staining for isocitrate dehydrogenase 1 mutation (IDH1 R132H) was negative in all 4 of the HG tumors in which it was performed. In addition, in retrospect, 1 anaplastic astrocytoma, grade III (tested 3/5 HG tumors) showed an H3 K27 M mutation, indicating a diffuse midline glioma, WHO grade IV. All HG tumors eventually showed enhancement, as did 5 of 7 (71.4\%) of the histologically confirmed LG tumors. Interestingly, secondary enhancement occurred at 189.0 months in 1 patient with initial diagnosis of diffuse astrocytoma, grade II, which subsequently progressed to glioblastoma, grade IV; no information on the ganglioglioma patient's time of enhancement was present in the record. The mean time to enhancement in the HG cohort was 87.0 months. Progression occurred in 3 of 7 (33.3\%) LG versus 4 of $5(80.0 \%) \mathrm{HG}$ tumors. The mean time to progression was 15.5 months for histologically diagnosed LG and 76.5 months for HG tumors. The patients' disease course and time points of biopsy/resection are summarized in Fig. 1.

\section{Quality of Life}

During routine clinical follow-up contacts, records for 12 patients (mean age 53.8 years) included questioning regarding quality of life. Two validated questionnaires for cancer patients were applied. EORTC-QLQ-C30 and EORTC-QLQ-BN20 scores (mean \%) are shown in Tables 2 and 3. Health-related quality-of-life scores were compared to scores for the healthy population, ${ }^{13}$ scores for glioma and metastatic brain cancer patients, $4,16,26$ and scores for long-term survivors of pediatric gliomas, which included WHO grade I (57\%) and II (43\%) tumors of various locations (Tables 2 and 3). ${ }^{22}$

In comparison to the healthy controls, tectal glioma patients had presented significant differences in scores for emotional functioning (mean 77.3 vs $32.2, \mathrm{p}<0.01$ ), cognitive functioning (75.8 vs $90.5, \mathrm{p}<0.01)$, social functioning (68.2 vs 31.5, p < 0.01) and financial difficulties (18.2 vs $5.7, \mathrm{p}<0.01$ ) (Tables 2 and 3). ${ }^{13}$ When compared to a general cohort of patients with newly diagnosed high-grade glioma ( $\mathrm{n}=745$, two phase III EORTC/NCIC trials) ${ }_{,}^{26}$ tectal glioma patients scored significantly better regarding future uncertainty $(18.8 \mathrm{vs} 37.0, \mathrm{p}<0.05)$, seizures $(0.0 \mathrm{vs}$ $60.1, \mathrm{p}<0.01)$ but worse for bladder dysfunction (36.0 vs $8.4, \mathrm{p}<0.01)$. This was confirmed in a smaller cohort of patients with newly diagnosed glioma by Klein and colleagues for future uncertainty $(18.8 \text { vs } 48.8, \mathrm{p}<0.01)^{16}$ and seizures $(0.0$ vs $18.6, \mathrm{p}<0.05)$ and additionally for visual disorders ( 7.5 vs $19.0, \mathrm{p}<0.05)$. Compared to scores from patients with brain metastases, scores for insomnia (18.2 

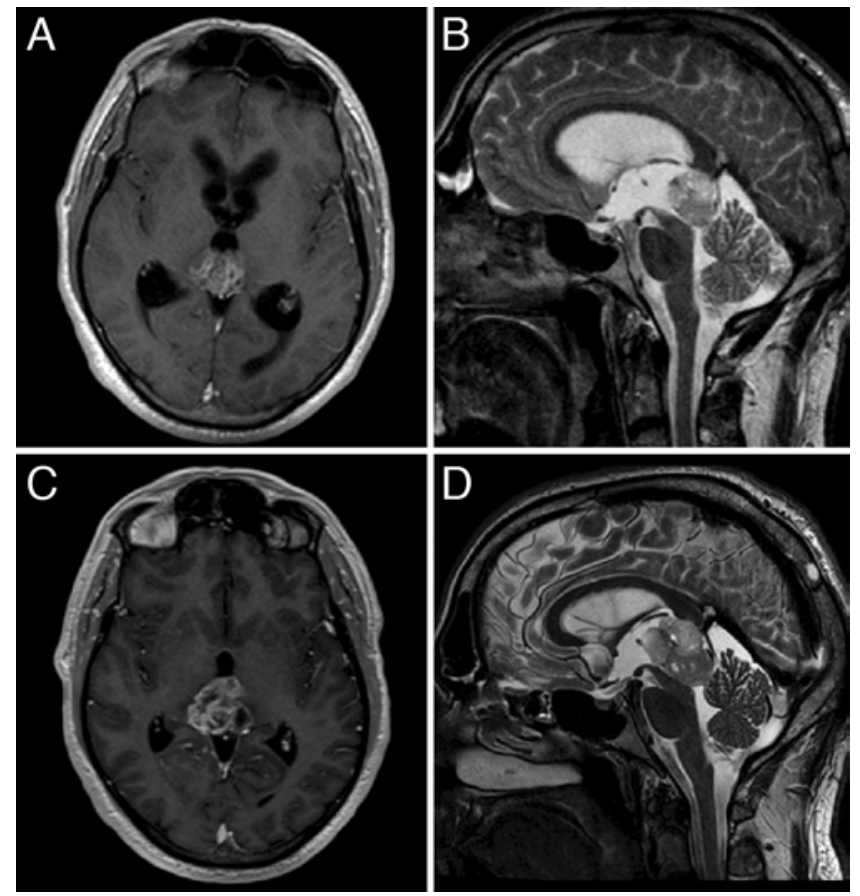

FIG. 2. Representative MR images. Available baseline (A and $B$ ) and short-term (1-month) follow-up (C and D) axial T1-weighted postcontrast ( $A$ and $C$ ) and sagittal T2-weighted ( $B$ and $D$ ) images obtained in an adult male patient with progressing tectal glioma (patient 4). The images shown here were obtained when the patient presented with progressive visual impairment, increasing cephalgia, and gait disturbance 15 years after initial diagnosis and stable disease.

vs $37.5, \mathrm{p}<0.05),{ }^{4}$ loss of appetite ( 0.0 vs $\left.19.5, \mathrm{p}<0.01\right)$, and bladder function (36.0 vs $11.1, \mathrm{p}<0.01)$ were significantly worse in our tectal glioma patients. In comparison to a cohort of long-term survivors of pediatric low-grade glioma, our tectal glioma patients scored significantly worse for social functioning (68.7 vs 87.0, $\mathrm{p}<0.05$ ), and drowsiness was more common $(37.9$ vs $20.0, p<0.05)$ in the tectal glioma patients, as was weakness of legs $(21.6$ vs $8.0, \mathrm{p}=0.05)$ and bladder dysfunction (36.0 vs 9.0, $\mathrm{p}<$ $0.01)$. The pediatric group had significantly lower scores for future uncertainty ( 0.0 vs $18.8, p<0.01)$.

\section{Discussion}

The rare prevalence and heterogeneous nature of tectal lesions has rendered the treatment debate of this entity controversial. Due to the small size of the tectal anatomical region, tectal gliomas represent a rare and heterogeneous pathology, as this report shows. Hence, no unique treatment approach has been defined, and most likely none even can be defined. In this context, we contribute our substantial series of patients diagnosed with intrinsic tectal gliomas, analyzed their clinical course, and discuss the highlighted potential for malignant transformation. Furthermore, since neuropsychological impairment represents a key parameter in the outcome of the more prevalent pediatric tectal glioma, we included a quality of life assessment to emphasize an additional clinical aspect of importance in this unique tumor entity.

Initial considerations of tectal lesions mainly concern

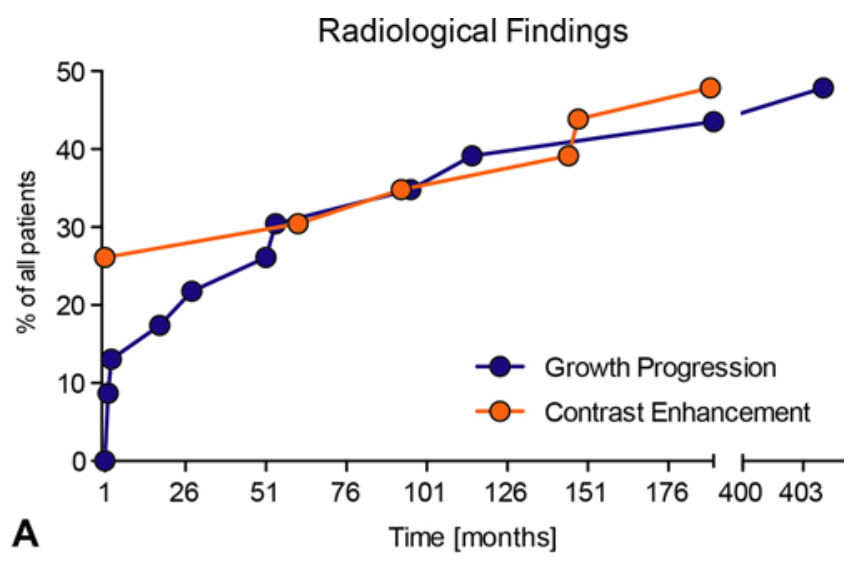

Time to Growth Progression

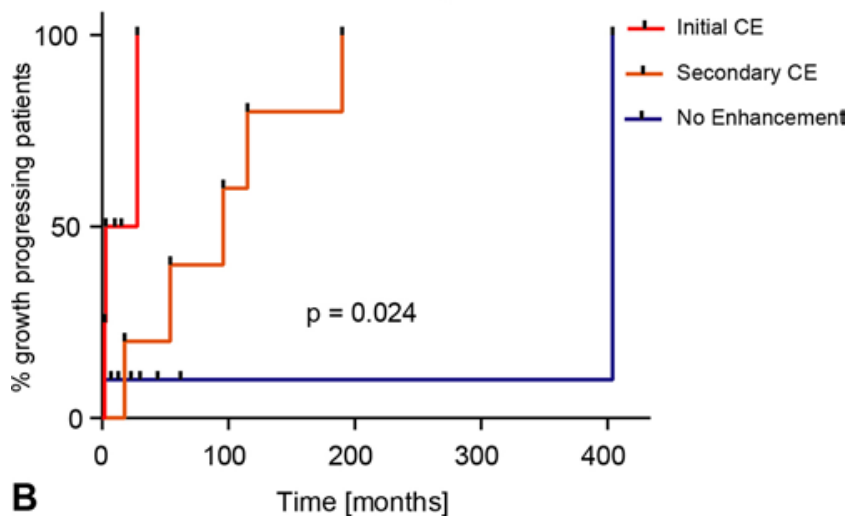

FIG. 3. A: Graphic depiction of the percentages of patients with tumor growth at follow-up and presence of contrast enhancement on imaging. B: Analysis of time to growth progression subdivided by time of contrast enhancement (CE) depicted as percentage of all patients whose tumors showed growth progression. Censored patients are indicated by black tick marks. Differences between the 3 groups were calculated by Mantel-Cox log-rank test $(p=0.024)$.

the management of hydrocephalus. As expected, due to their anatomical vicinity to the sylvian aqueduct the primary symptom leading to the initial diagnosis in our cohort was third ventricle hydrocephalus. It has been found acceptable to solely treat the hydrocephalus - using shunts or more recently endoscopic third ventriculostomy. $15,19,23,27$ Our series indicates that this may be sufficient for some patients, but a priori it cannot be expected that a tectal lesion will remain inert, as some smaller series have shown for pediatric patients. ${ }^{3,25}$ Within larger cohorts it has been found that tumors increase in size, leading to local compression, and also may show malignant degeneration requiring additional treatment to extend control and improve survival. ${ }^{11,20}$ After initial management of hydrocephalus, the diagnostic discussion focuses on the histological diagnosis. If hydrocephalus symptoms develop gradually and third ventriculostomy can be planned in a semi-elective time frame, concurrent biopsy can be performed. However, due to the histological heterogeneity of these tumors and the possibility of obtaining nonrepresentative areas, endoscopic biopsy can be considered inferior to stereotactic biopsies, which has demonstrated superior accuracy $(98 \%$ vs $78 \%){ }^{2}$ In addition, stereotactic biopsy demon- 







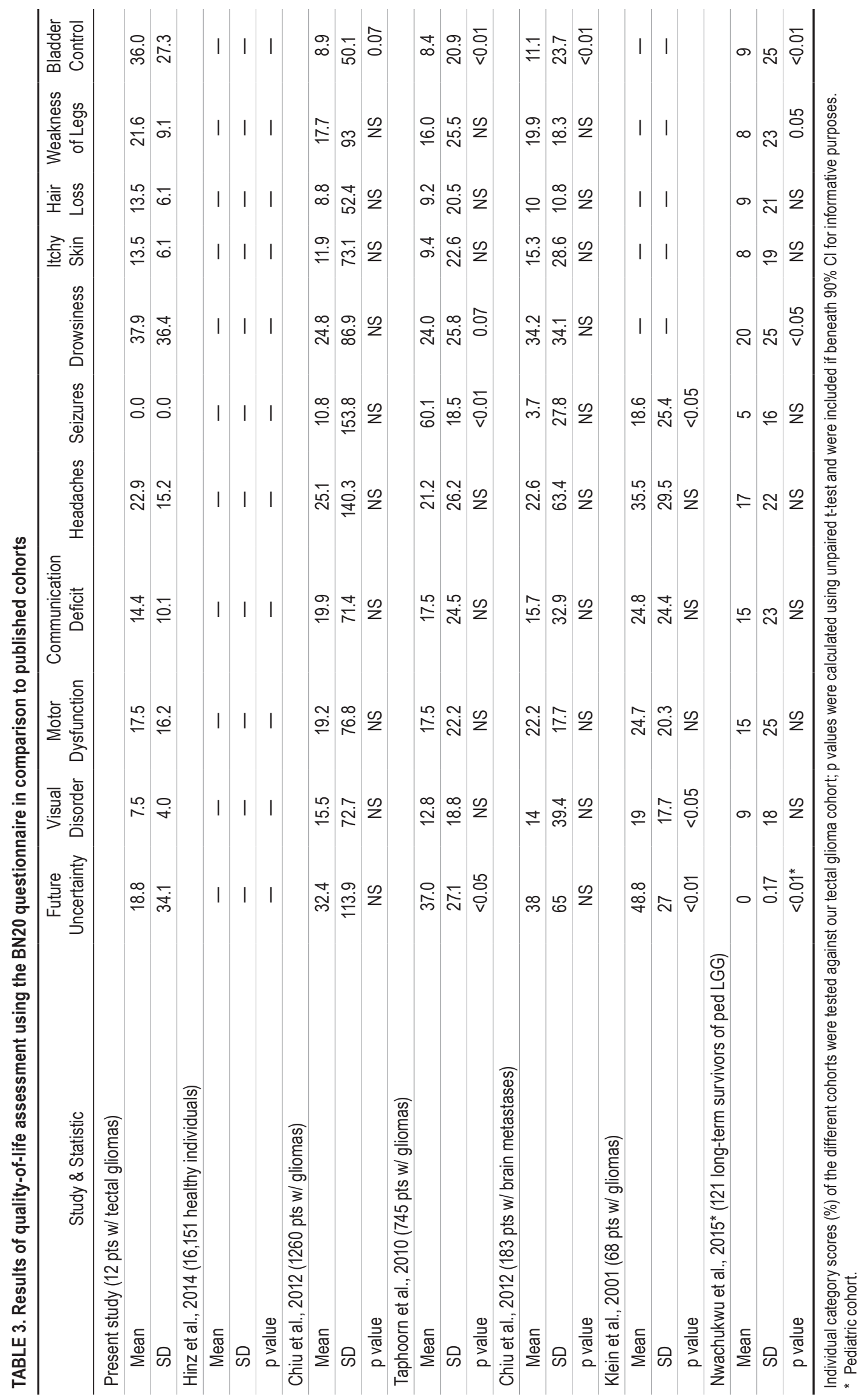


strated a trend toward a lower complication rate. ${ }^{1}$ Histological confirmation was pursued in $52.2 \%$ of patients in our cohort, and the rates of histological yield were $66.7 \%$ and $100 \%$ after the first and second biopsy, respectively. Although histological confirmation is desirable, invasive diagnostic can be postponed depending on the tumor's size and growth behavior, which will have to be assessed repetitively by MRI. If lesions appear suitable for resection, the open surgical approach achieves the most accurate diagnosis and will allow adjustment of the extent of resection according to the intraoperative results obtained from cryosections. To surgically access tectal lesions most surgeons prefer the same supracerebellar approach as for pineal lesions. ${ }^{21,24}$ Additional routes have been discussed, depending on the extent of the tumor. ${ }^{12,17}$ However, treating these lesions with aggressive surgery is controversial, and because of the location any surgical intervention is complex and harbors the risk for incapacitating complications. The most common complications include diplopia or upward gaze (Parinaud's syndrome) due to additional irritation of the quadrigeminal plate or postoperative pneumocephalus, if surgery was performed in the sitting position. ${ }^{6}$ Our case series demonstrates that, although postoperative symptoms were present, most were transient and did not result in longterm morbidity. Nevertheless, surgical removal of tectal lesions should be reserved for centers that have established sufficient neurosurgical expertise.

Tectal gliomas make up approximately $8 \%$ of all brainstem gliomas, reflecting the small mass of tissue. ${ }^{11}$ While brainstem gliomas have been described as progressive lesions with an unfavorable prognosis, ${ }^{11}$ tectal lesions are mostly considered benign and inert lesions possibly because of a different biology or an anatomical environment which is less critical than the dense long fiber tracts in the brain stem. Unfortunately, given the overall low prevalence and lack of larger case series, no clear histopathological diagnosis or pathophysiological basis was identified. Fisher et al. categorized into pilocytic and fibrillary astrocytoma for brainstem gliomas. ${ }^{7}$ In our series, about half of the histologically confirmed tumors were WHO grade I tumors and the other half demonstrated anaplastic de-differentiation (WHO grades III and IV). Interestingly, in our series higher-grade tumors presented less frequently with contrast enhancement at initial diagnosis, although this radiographic feature is associated with poor prognosis and early tumor progression as reproduced by our data. Although the tested high-grade tumors did not show positivity for IDH1 $\mathrm{R} 132 \mathrm{H}$ mutation, this finding points toward a progressive de-differentiation and evolution from lower-grade (WHO grade II) histology within patients with high-grade tumors. More importantly, we retrospectively identified 1 anaplastic astrocytoma (WHO grade III) as H3 K27 M mutated. This illustrates that the ability to perform molecular diagnostics could enhance the diagnostic precision of biopsies and increase the value of histological confirmation. Since most patients are monitored using repetitive longitudinal MRI, the prevalence of high-grade histologies is subject to bias, as patients typically undergo biopsy because of either suspicious contrast enhancement or growth progression. Nevertheless, 5 of $23(21.7 \%)$ patients presented with highgrade lesions - a significant proportion of patients with a malignant disease course. This raises the question of whether early biopsy and histological confirmation should be desirable for all tectal lesions and if MRI intervals, or even early treatment approaches, including radiotherapy, should be adopted depending on the histology.

Securing a definite diagnosis could also help patients to psychologically cope with their disease, as clinical experience has shown that patients with tectal gliomas suffer from tremendous psychological stress, which increases repeatedly with the approach of MRI appointments. This observation was confirmed in our quality-of-life assessment. Here, tectal glioma patients showed significant impairments in social, cognitive, and emotional functioning compared to healthy controls; ${ }^{13}$ in most cases, these differences were probably mediated by the overall psychological sequelae of the diagnosis and not the result of functional impairment caused directly by the tumor. This finding is supported by the fact that tectal glioma patients scored significantly better on questions related to insomnia and appetite loss compared to patients with metastasis, ${ }^{4}$ and also by the fact that they scored better on questions related to seizures than nontectal glioma patients did. ${ }^{16}$ The predominant somatic symptom in tectal glioma patients was drowsiness. Future uncertainty was documented in approximately $18 \%$ of interviewed patients. This finding can be interpreted controversially, as it is unclear how patients received and interpreted information on their prognosis, which in addition might be influenced by the widespread opinion of an overall indolent lesion. The findings of a prevalent malignant transformation of tectal lesions presented here should therefore be handled cautiously when providing information to patients and in the clinical management throughout follow-up, as the perception of an unpredictable threat can result in significant psychological morbidity in this patient cohort.

Our study was limited by the retrospective nature and due to the overall rare prevalence and clinical heterogeneity of this type of lesion. Individual assessments in the quality-of-life questionnaires were subject to bias, as not all patients were available to participate in this part of the study. In analogy, biopsies were predominantly taken from lesions that appeared large, suspicious, or progressive lesions on MRI, and therefore do not yield histological grading that is representative for all tectal lesions. Although the size of our cohort exceeds that of most published case series, the heterogeneity within the patient population does not allow for the postulation of general recommendations. Nevertheless, our observations demonstrate that malignant transformation is a prevalent feature of tectal gliomas. In this context, histological confirmation should be performed if feasible, as recommendations for radiological monitoring and psychological guidance during follow-up management can be affected by the tumor type.

\section{Conclusions}

This study finds tectal gliomas to be a relatively unpredictable and heterogeneous subgroup. Early and rapid clinical and radiographic progression do not always occur simultaneously, and detection of early signs of possible malignant transformation is crucial. Thus, performing a 
biopsy to obtain a histological diagnosis should be the rule rather than the exception-not least in the light of molecular pathology, emerging targeted therapies, and improved stereotactic radiation therapy.

\section{References}

1. Balossier A, Blond S, Touzet G, Lefranc M, de Saint-Denis T, Maurage CA, et al: Endoscopic versus stereotactic procedure for pineal tumour biopsies: Comparative review of the literature and learning from a 25-year experience. Neurochirurgie 61:146-154, 2015

2. Balossier A, Blond S, Reyns N: Endoscopic versus stereotactic procedure for pineal tumor biopsies: focus on overall efficacy rate. World Neurosurg 92:223-228, 2016

3. Bowers DC, Georgiades C, Aronson LJ, Carson BS, Weingart JD, Wharam MD, et al: Tectal gliomas: natural history of an indolent lesion in pediatric patients. Pediatr Neurosurg 32:24-29, 2000

4. Chiu L, Chiu N, Zeng L, Zhang L, Popovic M, Chow R, et al: Quality of life in patients with primary and metastatic brain cancer as reported in the literature using the EORTC QLQ-BN20 and QLQ-C30. Expert Rev Pharmacoecon Outcomes Res 12:831-837, 2012

5. Dağlioğlu E, Cataltepe O, Akalan N: Tectal gliomas in children: the implications for natural history and management strategy. Pediatr Neurosurg 38:223-231, 2003

6. Dallier F, Di Roio C: Sitting position for pineal surgery: Some anaesthetic considerations. Neurochirurgie 61:164167,2015

7. Fisher PG, Breiter SN, Carson BS, Wharam MD, Williams JA, Weingart JD, et al: A clinicopathologic reappraisal of brain stem tumor classification. Identification of pilocystic astrocytoma and fibrillary astrocytoma as distinct entities. Cancer 89:1569-1576, 2000

8. Gass D, Dewire M, Chow L, Rose SR, Lawson S, Stevenson C, et al: Pediatric tectal plate gliomas: a review of clinical outcomes, endocrinopathies, and neuropsychological sequelae. J Neurooncol 122:169-177, 2015

9. Griessenauer CJ, Rizk E, Miller JH, Hendrix P, Tubbs RS, Dias MS, et al: Pediatric tectal plate gliomas: clinical and radiological progression, MR imaging characteristics, and management of hydrocephalus. J Neurosurg Pediatr 13:1320,2014

10. Grimm SA, Chamberlain MC: Brainstem glioma: a review. Curr Neurol Neurosci Rep 13:346, 2013

11. Guillamo JS, Monjour A, Taillandier L, Devaux B, Varlet P, Haie-Meder C, et al: Brainstem gliomas in adults: prognostic factors and classification. Brain 124:2528-2539, 2001

12. Hernesniemi J, Romani R, Albayrak BS, Lehto H, Dashti R, Ramsey C III, et al: Microsurgical management of pineal region lesions: personal experience with 119 patients. Surg Neurol 70:576-583, 2008

13. Hinz A, Singer S, Brähler E: European reference values for the quality of life questionnaire EORTC QLQ-C30: Results of a German investigation and a summarizing analysis of six European general population normative studies. Acta Oncol 53:958-965, 2014

14. Igboechi C, Vaddiparti A, Sorenson EP, Rozzelle CJ, Tubbs RS, Loukas M: Tectal plate gliomas: a review. Childs Nerv Syst 29:1827-1833, 2013

15. Javadpour M, Mallucci C: The role of neuroendoscopy in the management of tectal gliomas. Childs Nerv Syst 20:852857,2004

16. Klein M, Taphoorn MJ, Heimans JJ, van der Ploeg HM, Vandertop WP, Smit EF, et al: Neurobehavioral status and health-related quality of life in newly diagnosed high-grade glioma patients. J Clin Oncol 19:4037-4047, 2001

17. Lapras C, Bognar L, Turjman F, Villanyi E, Mottolese C,
Fischer C, et al: Tectal plate gliomas. Part I: Microsurgery of the tectal plate gliomas. Acta Neurochir (Wien) 126:76-83, 1994

18. Lázaro BCR, Landeiro JA: Tectal plate tumors. Arq Neuropsiquiatr 64 (2B):432-436, 2006

19. Li KW, Roonprapunt C, Lawson HC, Abbott IR, Wisoff J, Epstein F, et al: Endoscopic third ventriculostomy for hydrocephalus associated with tectal gliomas. Neurosurg Focus 18(6A):E2, 2005

20. Matsuno A, Nagashima H, Ishii H, Iwamuro H, Nagashima $\mathrm{T}$ : Aggressive and invasive growth of tectal glioma after surgical intervention and chemoradiotherapy. Br J Neurosurg 20:246-249, 2006

21. Mottolese C, Szathmari A, Ricci-Franchi AC, Gallo P, Beuriat PA, Capone G: Supracerebellar infratentorial approach for pineal region tumors: Our surgical and technical considerations. Neurochirurgie 61:176-183, 2015

22. Nwachukwu CR, Youland RS, Chioreso C, Wetjen N, NageswaraRao A, Keating G, et al: Health related quality of life (HRQOL) in long-term survivors of pediatric low grade gliomas (LGGs). J Neurooncol 121:599-607, 2015

23. Oka K, Kin Y, Go Y, Hirakawa K, Tomonaga M, Inoue T, et al: Neuroendoscopic approach to tectal tumors: a consecutive series. Neurosurg Focus 6(4):e14, 1999

24. Pluchino F, Broggi G, Fornari M, Franzini A, Solero CL, Allegranza A: Surgical approach to pineal tumours. Acta Neurochir (Wien) 96:26-31, 1989

25. Robertson PL, Muraszko KM, Brunberg JA, Axtell RA, Dauser RC, Turrisi AT: Pediatric midbrain tumors: a benign subgroup of brainstem gliomas. Pediatr Neurosurg 22:6573, 1995

26. Taphoorn MJB, Claassens L, Aaronson NK, Coens C, Mauer M, Osoba D, et al: An international validation study of the EORTC brain cancer module (EORTC QLQ-BN20) for assessing health-related quality of life and symptoms in brain cancer patients. Eur J Cancer 46:1033-1040, 2010

27. Wellons JC III, Tubbs RS, Banks JT, Grabb B, Blount JP, Oakes WJ, et al: Long-term control of hydrocephalus via endoscopic third ventriculostomy in children with tectal plate gliomas. Neurosurgery 51:63-68, 2002

28. Westphal M, Emami P: Pineal lesions: a multidisciplinary challenge. Adv Tech Stand Neurosurg 42:79-102, 2015

\section{Disclosures}

The authors report no conflict of interest concerning the materials or methods used in this study or the findings specified in this paper.

\section{Author Contributions}

Conception and design: Martens, Mohme, Westphal. Acquisition of data: Martens, Mohme, Fritzsche, Löbel, Emami. Analysis and interpretation of data: Martens, Mohme, Fritzsche, Mende, Matschke, Löbel, Westphal. Drafting the article: Martens, Mohme, Fritzsche, Löbel, Westphal, Emami. Critically revising the article: Martens, Mohme, Fritzsche, Mende, Löbel, Westphal, Emami. Reviewed submitted version of manuscript: Martens, Mohme, Fritzsche, Matschke, Kammler, Westphal, Emami. Statistical analysis: Mende. Administrative/technical/material support: Martens, Mende, Matschke, Kammler, Westphal, Emami. Study supervision: Mohme, Westphal.

\section{Correspondence}

Tobias Martens: University Medical Center Hamburg-Eppendorf, Hamburg, Germany. t.martens@uke.de. 